r ScIDioc

\section{Alterations of Multipotent Mesenchymal Stromal Cells Induced by Interaction with Allogeneic Lymphocytes In Vitro}

Research Article

Kapranov NM${ }^{1}$, Davydova $\mathrm{YO}^{1}$, Galtseva IV ${ }^{1}$, Bakshinskayte $\mathrm{MV}^{2}$, Petinati NA ${ }^{1}$, Drize NJ ${ }^{1 *}$, Kuzmina LA ${ }^{1}$, Parovichnikova EN ${ }^{1}$, Savchenko VG

${ }^{1}$ Federal Government Budget Institution National Research Center for Hematology, Ministry of Health, Moscow, Russia.

${ }^{2}$ Biology Department, Sub-Department, Immunology, Moscow State University, Lomonosov, Russia.

\title{
Abstract
}

Multipotent mesenchymal stromal cells (MSCs) are widely used for cell therapy. Treatment with interferon- $\gamma$ (IFN $\gamma$ ) increases the immunomodulating properties of MSCs. When administered intravenously, MSCs interact with lymphocytes. It is impossible to follow the fate of MSCs in the recipient organism. The aim of this study was to investigate the properties of MSCs after their interaction with lymphocytes in vitro.

Bone marrow MSCs were co-cultured for 4 days with activated and non-activated lymphocytes. A portion of the MSCs was pretreated with IFN $\gamma$.

HLA-DR expression on the MSCs increased when these cells were co-cultured with lymphocytes and after they were treated with IFN $\gamma$. The activated lymphocytes induced significantly higher HLA-DR expression levels than did IFN $\gamma$ treatment. IFN $\gamma$ increased the viability of MSCs when these cells were co-cultured with lymphocytes. The immunomodulating properties of MSCs were amplified after IFN $\gamma$ priming and co-cultivation with lymphocytes; therefore, this amplification was not dependent on the IFN $\gamma$ source. IFN $\gamma$ treatment and lymphocyte interactions induced increases in the relative expression levels (RELs) of ICAM1 and factors involved in immunomodulation in the MSCs.

IFN $\gamma$ stabilizes MSCs while maintaining their viability. The data suggest that MSCs obtained from the hematopoietic cells donor or autologous should be used for cell therapy.

Keywords: Multipotent Mesenchymal Stromal Cells; Interferon $\gamma$-Lymphocytes; HLA-DR; gene Expression Level.

\section{Introduction}

Multipotent mesenchymal stromal cells (MSCs) are widely used in tissue engineering and cell therapy $[21,44]$. In tissue engineering, the ability of MSCs to differentiate into all tissues of mesenchymal origin is utilized. MSCs are mainly used for cell therapy due to their immune modulatory properties toward T-cells, B-cells, NK-cells, macrophages and dendritic cells $[4,49]$. MSCs affect Tcells via soluble immunosuppressive factors, such as transforming growth factor beta (TGF- $\beta$ ), hepatocyte growth factor (HGF), prostaglandin E synthase (PTGES), prostaglandin E2 (PGE2), human leukocyte antigen-G5 (HLA-G5), interleukin 10 (IL10), heme-oxygenase-1, indoleamine 2,3-dioxygenase (IDO1) [32], in- terleukin 6 (IL6) [28], stromal-derived factor 1 (SDF1), intercellular adhesion molecule 1 (ICAM1) [31], vascular cell adhesion molecule 1 (VCAM1) [7], matrix metalloproteinases (MMP) [52], and others [55]. MSCs engage in direct intercellular interactions with lymphocytes via PD-L1/PD-1, CD200/CD200R, FAS-L/ FAS, the costimulatory molecules $\mathrm{B} 7-\mathrm{H} 4$ and toll-like receptors $[1,35,38,50,63]$.

Due to their immunomodulatory properties, MSCs are used for the treatment of acute graft versus host disease (aGVHD), autoimmune diseases, inflammatory bowel diseases, and diabetes mellitus [29, 60]. However, the use of MSCs is not always effective. According to some studies, the immunoregulatory potential

*Corresponding Author

Drize Nina PhD,

FGBI National Research Center for Hematology, ZykovskiyNovyy pr., 4, Moscow, 125167, Russia.

Email: ndrize@yandex.ru

Received: April 27, 2017

Accepted: June 05, 2017

Published: June 08, 2017

Citation: Kapranov NM, Davydova YO, Galtseva IV, Bakshinskavte MV, Drize NJ, et al., (2017) Alterations of Multipotent Mesenchymal Stromal Cells Induced by Interaction with Allogeneic Lymphocytes In Vitro. Int J Stem Cell Res Transplant. 5(2), 277-286. doi: http://dx.doi.org/10.19070/2328-3548-1700042

Copyright: Drize NJ ${ }^{\circ}$ 2017. This is an open-access article distributed under the terms of the Creative Commons Attribution License, which permits unrestricted use, distribution and reproduction in any medium, provided the original author and source are credited. 
of MSCs increases after their activation, and these cells become more effective in suppressing the immune response. It is expected that some molecules can be used for MSC activation. Currently the possibility of activating MSCs with various substances, such as IFN- $\gamma$, TNF- $\alpha$, and IL17, is being investigated [10, 41]. It has been shown that IFN- $\gamma$ is needed for the manifestation of the immunosuppressive properties of MSCs: MSCs do not have an effect on the proliferation of IFN- $\boldsymbol{\gamma}$-deficient T-cells [50]. MSCs activated by IFN- $\gamma$ (MSCs- $\gamma$ ) have an increased anti-inflammatory effect, and blocking the receptors for IFN- $\gamma$ leads to a significant decrease in immunosuppression [52]. In contrast, pretreatment of MSCs with IFN- $\gamma$ leads to an increase in their immunomodulatory properties [42]. However, MSCs- $\gamma$ became more immunogenic due to an increase in the expression of MHC-I and the appearance of MHC-II on the cell surface [11]. After MSCs interact with IFN- $\gamma$, they began to produce high levels of IDO1, soluble enzyme that cleaves tryptophan, resulting in the accumulation of kynurenine, which has immunosuppressive properties. The efficiency of the IFN- $\gamma$ treatment depends on its concentration since IFN- $\gamma$ does not change the immunomodulatory properties of MSCs at a low concentration $(50 \mathrm{U} / \mathrm{ml})$, whereas it enhances the immunosuppressive properties of MSCs at a high concentration $(500 \mathrm{U} / \mathrm{ml})$ $[32,40]$. The activated MSCs show increased expression levels of IL6 and IDO1, leading to more efficient inhibition of T-cell and NK-cell proliferation $[20,36]$. MSCs with enhanced IDO1 secretion can more effectively suppress the T-cell-mediated immune response by inhibiting proliferation and inducing apoptosis in $\mathrm{T}$ cells. MSCs- $\gamma$ boost the number of regulatory T-cells, which may be partly due to increased expression of PD-L1 [52].Additionally, the activated MSCs are characterized by more pronounced suppression of CD25 expression on CD4+ effector T-cells and the suppression of NK-cells compared to non-activated MSCs. The expression levels of IFN- $\gamma$, TNF- $\alpha$ and IL2 decrease in MSCs- $\gamma$, but the expression level of PGE2 does not change, which also contributes to the immunomodulatory properties of these cells. MSCs do not express the co-stimulatory molecules CD80, CD83, and CD86 even under the influence of IFN- $\gamma$ [53], and CD40 expression data are contradictory $[8,53]$. After MSCs are primed by IFN- $\gamma$, they begin to express MHC II molecules that promote Tcell activation and cytotoxic reactions. Clinical use of MSCs- $\gamma$ can lead to complications including acute immune responses. The application of MSCs- $\gamma$ in animal models has been effective. Crohn's disease has been modeled in mice that have been administered dextran coupled with trinitrobenzene sulfonate. Administration of human MSCs- $\gamma$ in these mice led to better survival, and the mice gained weight as the severe symptoms of colitis subsided [17]. Thus, the ability to increase the therapeutic efficacy of MSCs has been illustrated.

MSCs have been well characterized in vitro. However, what happens to MSCs after intravenous injection remains unclear. It is known that 2 weeks after infusion, MSCs could not be detected in the recipient organism [2]. Typically, MSCs are administered intravenously, and their interaction with lymphocytes occurs in the blood and tissues. MSCs express multiple adhesion molecules on their surface, which allows them to interact with lymphocytes [5]. Lymphocytes secrete cytokines that act on the MSCs [22]. As a result, properties of the MSCs, such as their ability to modulate the immune response and their trophic function, can change. The immune system of the recipient thus gains the ability to recognize these foreign MSCs, and the MSCs lose their immune privilege
$[3]$.

The aim of this study was to investigate the changes in the main properties of MSCs after these cells interact with allogeneic lymphocytes. The MSCs were co-cultured with lymphocytes for 4 days, and their basic properties were analyzed over time. The interaction of MSCs with lymphocytes led to the appearance of HLA-DR expression on the surface of MSCs. CD90 expression was reduced over time, and IDO1, CFH, PTGES, IL6, and CSF1 expression was elevated. These changes may affect the duration of exogenous MSC survival in the recipient organism.

\section{Materials And Methods}

\section{MSCs}

MSCs were isolated from the bone marrow of 13 donors $(7$ males and 6 females), ranging in age from 22 to 62 years (median: 27 years). After informed consent was obtained, samples were collected during the aspiration of hematopoietic stem cells for allogeneic transplantation at the Department of Bone Marrow Transplantation. The protocol was approved by the local medical ethics committee.

The MSCs were derived from 5-10 $\mathrm{ml}$ of donor bone marrow. For the separation of mononuclear cells, the bone marrow was mixed with an equal volume of alpha-MEM (ICN) containing $0.2 \%$ methylcellulose (1500 cP, Sigma-Aldrich). After $40 \mathrm{~min}$, most of the erythrocytes and granulocytes had precipitated, while the mononuclear cells remained in suspension. The suspended (upper) fraction was aspirated and centrifuged for $10 \mathrm{~min}$ at $450 \times \mathrm{g}$.

The cells from the sediment were re-suspended in a standard culture medium that was composed of alpha-MEM supplemented with 10\% fetal bovine serum (Hy Clone), $2 \mathrm{mM}$ L-glutamine (ICN), $100 \mathrm{U} / \mathrm{ml}$ penicillin (Ferein) and $50 \mathrm{microg} / \mathrm{ml}$ streptomycin (Ferein). The cells $\left(3 \times 10^{6}\right)$ were cultured in T25 culture flasks (Corning-Costar). When a confluent monolayer of cells had formed, the cells were washed with $0.02 \%$ EDTA (ICN) in a physiological solution (Sigma-Aldrich) and then trypsinized (ICN). The cells were seeded at $4 \times 10^{3}$ cells per $\mathrm{cm}^{2}$ of flask growth area. The cultures were maintained at $37^{\circ} \mathrm{C}$ in $5 \% \mathrm{CO}_{2}$. The number of harvested cells was counted directly; cell viability was checked by trypan blue dye exclusion staining.

All MSCs were immunophenotyped with following markers: CD105, CD73, CD45, CD34, CD14, HLA-DR using standard protocols. Antibodies were purchased at BD Pharmingen (CD105, CD59, CD73, CD90, CD31, CD34 and CD14), Sigma (CD45, FSP) and DAKO (HLA-DR). All MSCs were in accordance with MSCs minimal definition criteria [16].

Human interferon gamma (IFN- $\gamma$ ) (Ingaron, Farmaclone) was dissolved in alpha-MEM and added at a concentration of $500 \mathrm{U} /$ $\mathrm{ml}$ to the MSC culture for 4 hours. Then, the culture medium was changed [40].

\section{RNA}

Total RNA was extracted from the MSCs at passage 1 by the standard method [13], and cDNA was synthesized using a mixture 
of random hexamers and oligo (dT) primers. Gene expression levels were quantified by real-time quantitative PCR using hydrolysis probes (TaqMan) and an ABI Prism 7500 (Life Technologies). Gene-specific primers were designed by the authors and synthesized by Syntol R\&D. All primers and probe sequences are provided in Supplement 1. The relative expression levels (RELs) of the genes were determined by normalizing the expression of each target gene to the levels of $\beta A C T I N$ and GAPDH and calculated using the $\Delta \Delta C_{t}$ method [48] for each MSC sample.

\section{Immunophenotyping}

The expression of the MHC antigens on the MSC level was determined by flow cytometry. After the MSCs were removed from the bottom of the flask, they were washed twice with Cell WASH (BD Biosciences, USA), and then $2 \times 10^{4}$ cells were incubated for $20 \mathrm{~min}$ in the dark with one of the following monoclonal antibodies: anti-CD90 labeled with PE (BD Pharmingen, USA), antiCD54 labeled with APC (BD Biosciences, USA) or anti-HLA-DR labeled with APC (BioLegend, USA). Analyses were performed using a FACS Canto II flow cytometer (BD Biosciences, USA), and the data were analyzed using FACS Diva software (BD Biosciences, USA).

The MSC population was first determined by forward and side scattering parameters and then isolated based on the expression of CD90. For this cell population, the mean fluorescence intensities (MFIs) for the channels PE (CD90) and APC (HLA-DR) were evaluated.

\section{Lymphocytes preparation}

For the analysis of interactions between MSCs and lymphocytes, lymphocytes from healthy donors were separated using Lymphoprep solution at a density of $1.077 \mathrm{~g} / \mathrm{cm}^{3}$ (MP Biomedicals). The obtained mononuclear cell fraction was washed 3 times with RPMI-1640 medium without serum. All the experiments were performed on lymphocytes from the same donor. In some experiments, lymphocytes derived from MSCs from the donor's peripheral blood were used in addition to the standard. Lymphocytes and MSCs were co-cultured for 4 days at $37^{\circ} \mathrm{C}$ and $5 \% \mathrm{CO}_{2}$. As controls, lymphocytes cultured without MSCs were used.

\section{Schedule of experiments}

MSCs at 2-3 passages were seeded at a density of $10^{5}$ cells per T25 flask, and a day later, some of the flasks were treated with IFN- $\gamma$ for 4 hours (MSCs- $\gamma$ ). Then, the flasks were washed and $10^{6} \mathrm{lym}-$ phocytes suspended in RPMI-1640 medium supplemented with $10 \%$ FBS were added. In some experiments, the lymphocytes were activated with $5 \mathrm{mg} / \mathrm{ml}$ phytohemagglutinin (PHA-lymphocytes). MSCs and MSCs- $\gamma$ cultured in RPMI-1640 medium without lymphocytes were used as controls. Flasks were kept for 4 days after the addition of the lymphocytes at $37^{\circ} \mathrm{C}$ and $5 \% \mathrm{CO}_{2}$.

After the $1^{\text {st }}, 2^{\text {nd }}, 3^{\text {rd }}$ and $4^{\text {th }}$ days, the lymphocytes were washed from the MSC layers. The MSCs were removed by trypsinization, and the number of viable cells was determined by the dye exclusion method (trypan blue). For each MSC culture, flow cytometric analysis was performed as described above.

\section{Statistics}

The data are presented as the means \pm SEM. The data were analyzed using Student's $t$-test (considered reliable at $\mathrm{p}<0.05$ ).

\section{Results And Discussion}

After intravenous administration, MSCs interact with lymphocytes in the peripheral blood and various tissues. An attempt to study the dynamics of changes in the characteristics of MSCs following their interaction with lymphocytes in vitro was made. Activated and non-activated allogeneic lymphocytes were explanted onto MSC cultures at a ratio of approximately 10:1 with RPMI1640 medium with $10 \%$ FBS. This ratio of lymphocytes to MSCs adequately reflects the situation after intravenous administration of MSCs to prevent or treat a GVHD after the transplantation of allogeneic hematopoietic stem cells $[27,47]$. Some samples of MSCs were pretreated with IFN- $\gamma$ for 4 hours. The scheme of the experiments is shown in Figure 1.

MSCs are considered immune privileged because they do not express HLA-DR on their surface in vitro.

Figure 1. Scheme of Experiments.

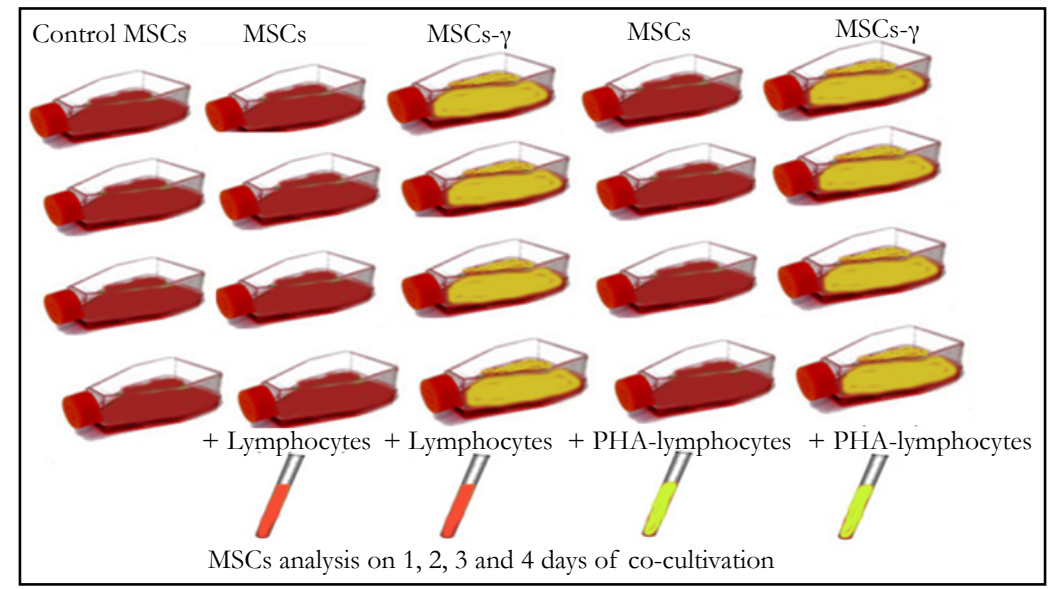

MSCs were seeded to 4 flasks for each variant. Lymphocytes or PHA-Lymphocytes were added one day after culture establishment.

Then each day 1 flask per variant was analyzed. 
HLA-DR expression of on the surface of MSCs after these cells interact with lymphocytes

In the MSCs co-cultured with both autologous and allogeneic lymphocytes, the level of HLA-DR surface expression gradually increased by 1.4 times on day 1 and then by $1.7,1.7$ and 2 times $(p=0.03$ ) on days 2, 3 and 4, respectively (Figure 2A, B). It is known that an increase in HLA-DR expression on MSCs can occur after their treatment with immunomodulatory agents without T-cell activation [14].

When MSCs were co-cultured with PHA-lymphocytes, the level of HLA-DR expression on these cells increased more significantly than when they were co-cultured with non-activated lymphocytes, with increases of 2.8 times after the 1 st day and then 9.1, 12.2 and 12.3 times after the $2 \mathrm{nd}$, 3rd, and 4th day, respectively $(\mathrm{p}<0.05$ in all cases)(Figure $2 \mathrm{C}$ ). IFN $-\gamma$ priming caused an increase in the level of HLA-DR expression on MSCs- $\gamma$. This expression of HLA-DR on the MSCs- $\gamma$ was more pronounced than in the presence of non-activated lymphocytes. HLA-DR expression on the MSCs- $\gamma$ without lymphocyte interaction increased 2.2 times after $1^{\text {st }}$ day $(p=0.009)$ and $4.7(p=0.001)$ and $6.3(p=0.0004)$ and $8.2(p=0.001)$ times on each subsequent day (Figure 2D). The MSCs- $\gamma$ co-cultured with lymphocytes showed a much greater increase in HLA-DR expression than MSCs that interacted with lymphocytes: an increase of 2.4 times after the $1^{\text {st }}$ day and increases of 5.0, 5.5 and 4.6 times after the $2^{\text {nd }}, 3^{\text {rd }}$, and $4^{\text {th }}$ day, respectively $(\mathrm{p}<0.05$ in all cases) (Figure 2E). However, this increase was not as pronounced as after the MSCs- $\gamma$ interacted with PHA-lymphocytes (Figure 2F). It was found that after 4 days of co-culturing of MSCs- $\gamma$ with lymphocytes, the level of HLA-DR expression was significantly lower $(p=0.05)$ than in the MSCs- $\gamma$ without lymphocytes. Co-culturing of MSCs- $\gamma$ with
PHA-lymphocytes resulted in HLA-DR expression increases of 3.6, 11.9, 13.7 and 12.4 times during each subsequent day, and this result did not differ from that seen in the MSCs (Figure 2F). Previously, it has been shown that MSCs- $\gamma$ have a phenotype similar to that of MSCs (expression of markers such as CD45, CD11b, CD44 and CD29 [40]), which is consistent with our observation.

Thus, after intravenous administration of MSCs or MSCs- $\gamma$ and the interaction of these cells with lymphocytes, the HLA-DR expression level would most likely increase in the same manner, i.e., the activation of MSCs with IFN- $\gamma$ will not significantly affect the immune response in vivo.

The study of MSC growth parameters in the presence of lymphocytes showed a significant increase in the proportion of dead cells. It is known that foreign MSCs are not detected in the recipient 2 weeks after intravenous administration $[2,19]$. The data obtained can partly explain these observations.

\section{MSC growth parameters}

When MSCs were cultured in aMEM supplemented with $10 \%$ FBS, their numbers increased 2-3 times within 3-4 days after passaging [56]. In these experiments, the MSCs were cultured in RPMI-1640 with 10\% FBS, which resulted in a reduction in the proliferation of these cells. Even in the control samples, adequate increases in the cell number were not observed. Cellular production did not change significantly in MSCs- $\gamma$ during co-culture with lymphocytes (Table 1). When MSCs were co-cultured with PHAlymphocytes, their cell number decreased regardless of IFN- $\gamma$ pretreatment; however, these changes were not significant.

The proportion of living cells in the cultures varied, and pretreat-

Figure 2. Dynamics of HLA-DR Expression on MSCs.

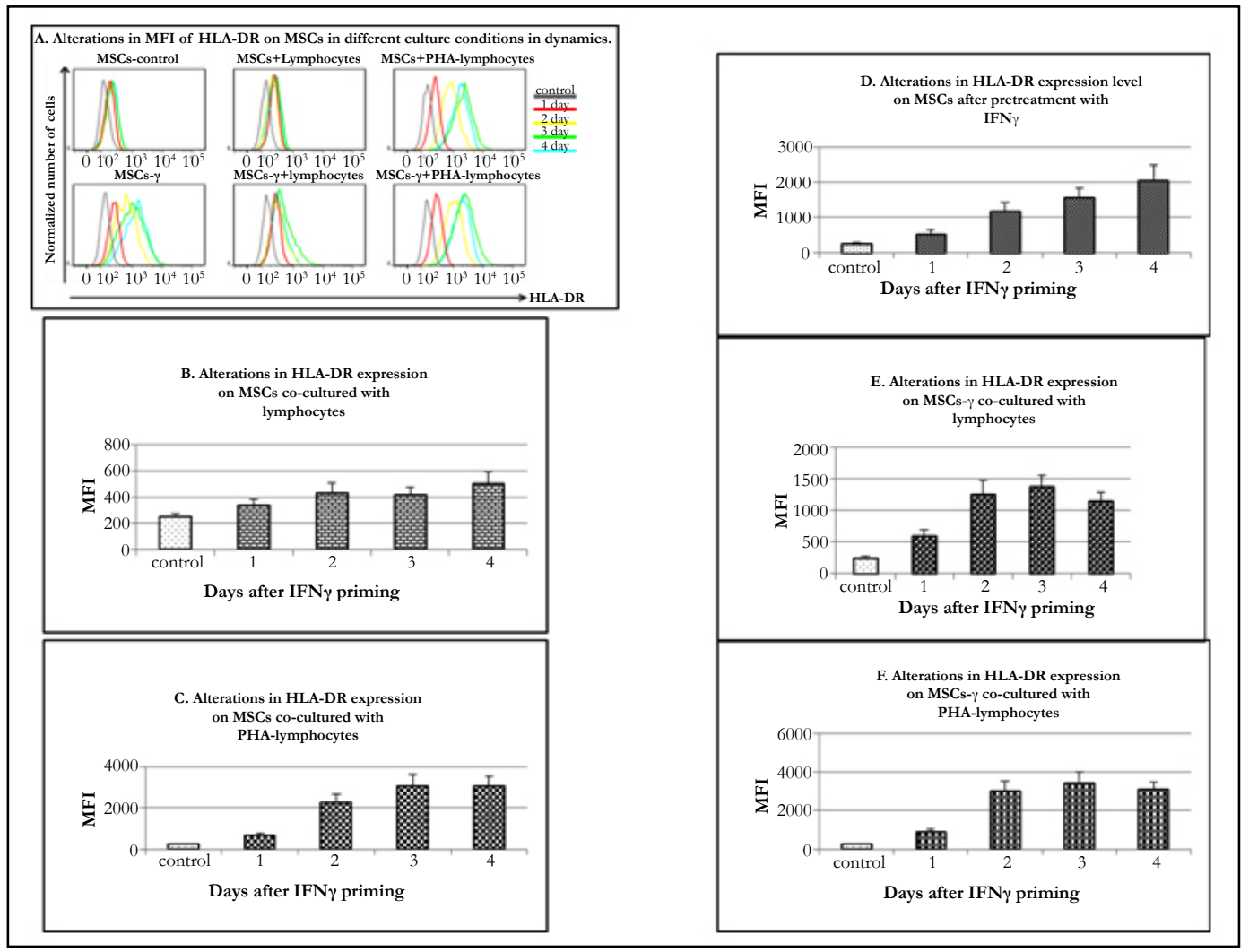


ment with IFN- $\gamma$ led to an insignificant decrease in the proportion of viable cells. A significant decrease in the proportion of viable MSCs was revealed in co-cultures with PHA-lymphocytes (Figure 3.) after 4 days, while such an effect was not observed in cultures pretreated with IFN- $\gamma$. This finding may indicate the sensitization of MSCs- $\gamma$ to factors secreted by the activated lymphocytes.

The differentiation potential of MSCs can change after injection. It is known that the expression of CD90 (a MSC markers)decreases during the differentiation of these cells [34, 51]. Furthermore, Thy-1 (CD90) glycoproteins are involved in cell-cell interactions. The study of CD90 expression on the MSCs co-cultured with lymphocytes showed a decrease in its expression levels.

\section{CD90 expression on MSCs}

The CD90 expression levels on MSCs and MSCs- $\gamma$ did not change during culture. On the MSCs and MSCs- $\gamma$ co-cultured with lymphocytes, the CD90 expression level gradually decreased: after 2 days, it differed from the original, and by the $4^{\text {th }}$ day, it decreased by 1.5 times (Figure 4A, B, C). Co-culture of MSCs and MSCs- $\gamma$ with PHA-lymphocytes resulted in a more significant reduction in the CD90 expression level (1.3, 1.2, 1.5, 2.3 and 1.1, 1.0, 1.6, and 2.1 times after the $1^{\text {st }}, 2^{\text {nd }}, 3^{\text {td }}$ and $4^{\text {th }}$ day, respectively) (Figure $4 \mathrm{D}$, E). The findings indicate that the interaction with lymphocytes lead to MSC differentiation.

To test this hypothesis, the expression of the standard differentiation markers of MSCs was investigated. The genes involved in osteogenic differentiation (SPP1 and BGLAP), adipogenic differentiation (PPARG) and chondrogenic differentiation (SOX9) were analyzed. The relative expression levels (RELs) of SOX9 and BGLAP did not change in any of the experiments. The REL of SPP1 increased by 2 times in the MSCs- $\gamma$ co-cultured with lymphocytes $(\mathrm{p}=0.04)$.

PPAR- $\gamma$ is one of the key regulators of adipogenesis. In the absence of PPAR- $\gamma$, there is no formation of normal adipocytes [25]. The REL of PPARG did not change in the MSCs during culture. In the MSCs- $\gamma$, the REL of PPARG increased more than 2 times $(1.1 \pm 0.4$ on the first day compared to $2.7 \pm 0.8$ on day 4 , $\mathrm{p}=0.04)$. It is important to note that one of the main functions of
PPAR- $\gamma$, as a member of the nuclear receptor family, is the regulation of gene expression at the mRNA level. PPAR- $\gamma$ regulates chemokine activation after treatment with IFN- $\gamma$ [54].

After 4 days of MSCs and MSCs- $\gamma$ co-culture with lymphocytes, the REL of PPARG significantly decreased from $1.4 \pm 0.4$ to 0.5 $\pm 0.1(p=0.04)$ and from $2.7 \pm 0.8$ to $0.9 \pm 0.3(p=0.02)$, respectively. Opposite results were obtained by Wu et al., [62]. When the MSCs were co-cultured with PHA-lymphocytes in this study, the REL of PPARG significantly increased. The analysis in that paper was carried out one day after the beginning of incubation. In our study, no significant differences were detected on the first day. A significant increase in the REL of PPARG was detected only in the IFN- $\gamma$-primed MSCs without lymphocytes after 4 days of incubation. Perhaps these contrasting results are due to the significant differences in the ratios of lymphocytes and MSCs in these studies. It is known that PPAR- $\gamma$ is expressed mainly in adipose tissue cells, but it is also expressed in lymphocytes, macrophages, fibroblasts and chondrocytes [37]. In addition, the increase in PPARG expression could be attributed to an additive effect, which we did not observe in our experiments.

Thus, the interaction of MSCs with lymphocytes have different influences on the differentiation potential of MSCs. It is possible that IFN- $\gamma$ priming changes the osteogenic and adipogenic differentiation abilities of MSCs but not their chondrogenic differentiation ability.

Reduced CD90 expression affects the intercellular interactions and adhesion of activated T-cells $[26,46]$. This interaction is supported by integrins. In addition to CD90, the cell adhesion molecule ICAM-1 is important in the study of MSC interactions with lymphocytes. ICAM-1 is a ligand of the LFA- 1 integrin receptor, which is detectable on leukocytes and whose activation is necessary for the transition of lymphocytes into the tissue [64].

\section{ICAM-1}

ICAM-1 is present at low concentrations on the membranes of leukocytes and endothelial cells [59]. According to our immunofluorescence studies, $76.4 \pm 1.3 \%$ of the MSCs expressed ICAM1 (CD54). When the MSCs were co-cultured with lymphocytes

Figure 3. Alterations in the Proportion of Viable Cells in Culture.

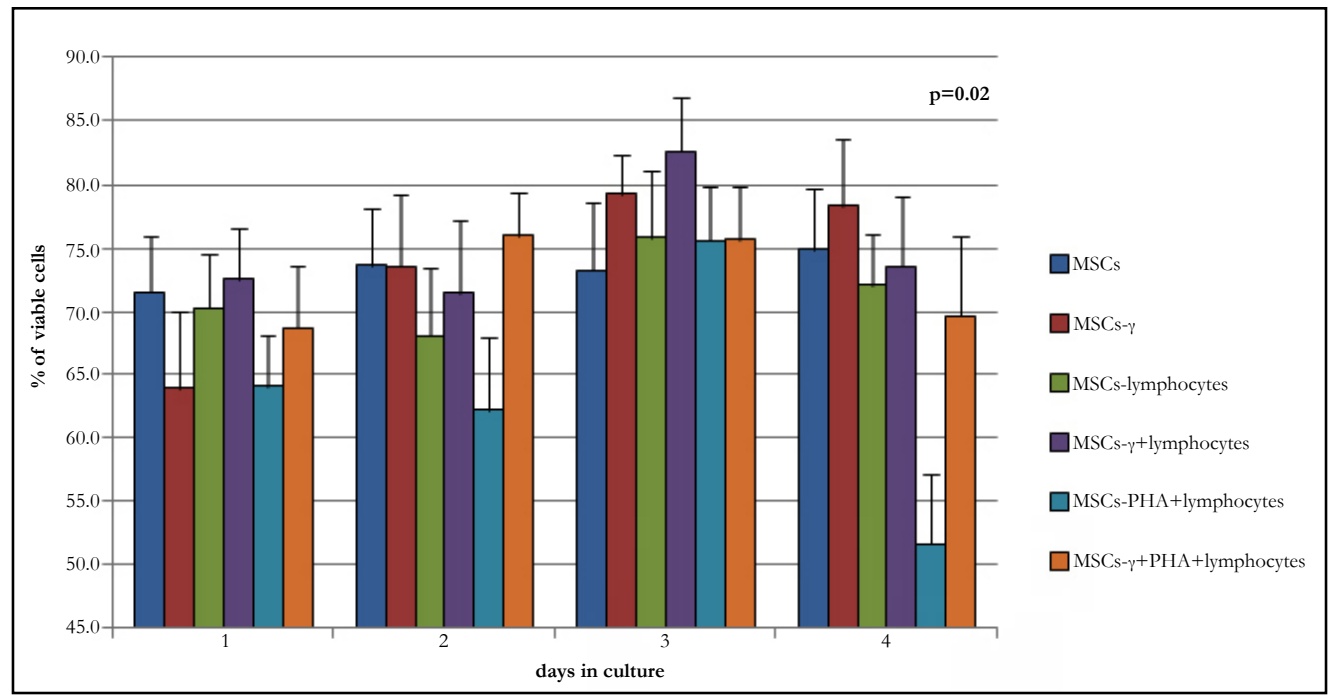


Figure 4. Dynamics of CD90 expression on MSCs.

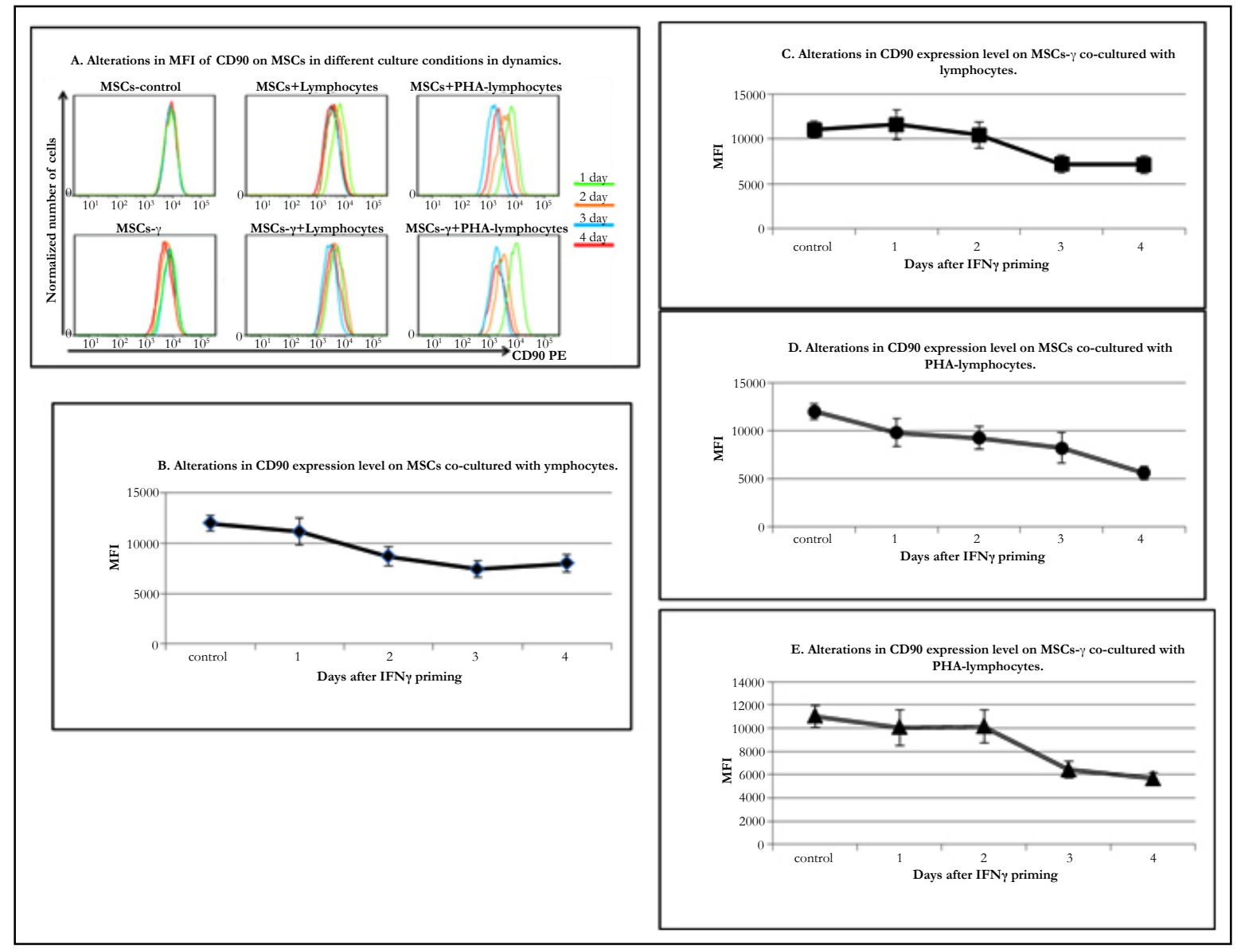

and PHA-lymphocytes, ICAM-1 began to be expressed on all MSCs, whereas the REL of ICAM-1, as determined by RT-PCR, increased 8 times and 35.1 times, respectively (Figure 5.). It is known that MSC stimulation by cytokines, including IL-1 and TNF- $\alpha$, sharply increases ICAM-1 expression on the cytoplasmic membrane $[6,15]$. After MSC pretreatment with IFN- $\gamma$, the proportion of cells expressing ICAM-1 was significantly increased $(\mathrm{p}=0.008)$ by up to $89.8 \pm 1.8 \%$. Gene expression analysis revealed that the REL of ICAM-1 in the MSCs- $\gamma$ increased significantly by 3.6 times,14.6 times when co-cultured with lymphocytes and 49.8 times with PHA-lymphocytes (Figure 5.) after 4 days of co-culturing. It is known that co-culture of MSCs with neutrophils also increases ICAM-1 expression levels by up to 100 times compared to controls [23]. Increased ICAM-1 expression after treatment with IFN- $\gamma$ is in agreement with the data from other researchers [45].

Increased ICAM-1 expression enhances cell-to-cell interactions. MSCs are the elements of the hematopoietic stromal microenvironment that regulate the process of hematopoiesis locally by cell-to-cell interactions and distantly through secreted soluble factors [18]. MSCs administered intravenously effectively participate in immunomodulation due to cell-cell contacts, which are enhanced by increasing the expression of ICAM-1, and due toparacrine functions and microvesicles $[9,55]$.

The immunomodulatory agents of MSCs include IDO1, PTGES, and CFH, as well as other chemokines and cytokines [24].

Interaction with lymphocytes and pretreatment with IFN- $\gamma$ sig- nificantly increase the expression of IDO1 in MSCs.

\section{IDO1 expression}

The REL of IDO1 increased dramatically in MSCs after both IFN- $\gamma$ pretreatment and lymphocyte co-culture (Figure 6A). When the MSCs were co-cultured with lymphocytes, the REL of IDO1 increased by 250 times, but by the $4^{\text {th }}$ day of culture, it gradually decreased by 4 times (Figure 6C). The REL of IDO1 in MSCs- $\gamma$ increased by 350 times compared with control MSCs; however, on the $4^{\text {th }}$ day of culture, there was only a 10 -fold increase in the REL of IDO1 compared to controls (Figure 6B). The addition of lymphocytes to the MSCs- $\gamma$ increased the REL of IDO1 by 750 times, and it decreased by only 2 times after 4 days of co-culture (Figure 6D). When MSCs and MSCs- $\gamma$ were co-cultured with PHA-lymphocytes, the REL of IDO1 increased by 2000-4000 times, and there was no reduction in this level during culture (Figure 6E, F).

Co-culture of MSCs with lymphocytes and their priming by IFN- $\gamma$ significantly increased the REL of IDO1. When lymphocytes were co-cultured with MSCs, they begin to express high levels of IFN- $\gamma$. The expression of IFN- $\gamma$ by lymphocytes greatly increases after their activation with PHA [58]. The dual effect of IFN- $\gamma$ and lymphocytes induced a similar dramatic increase in the REL of IDO1. These data correlate with the results obtained by Zhang and co-authors [65]. In that work, the escalation of IFN- $\gamma$ doses increased the expression of IDO1 at both the RNA and protein levels. 
Figure 5. Alterations in REL of ICAM1 in MSCs.

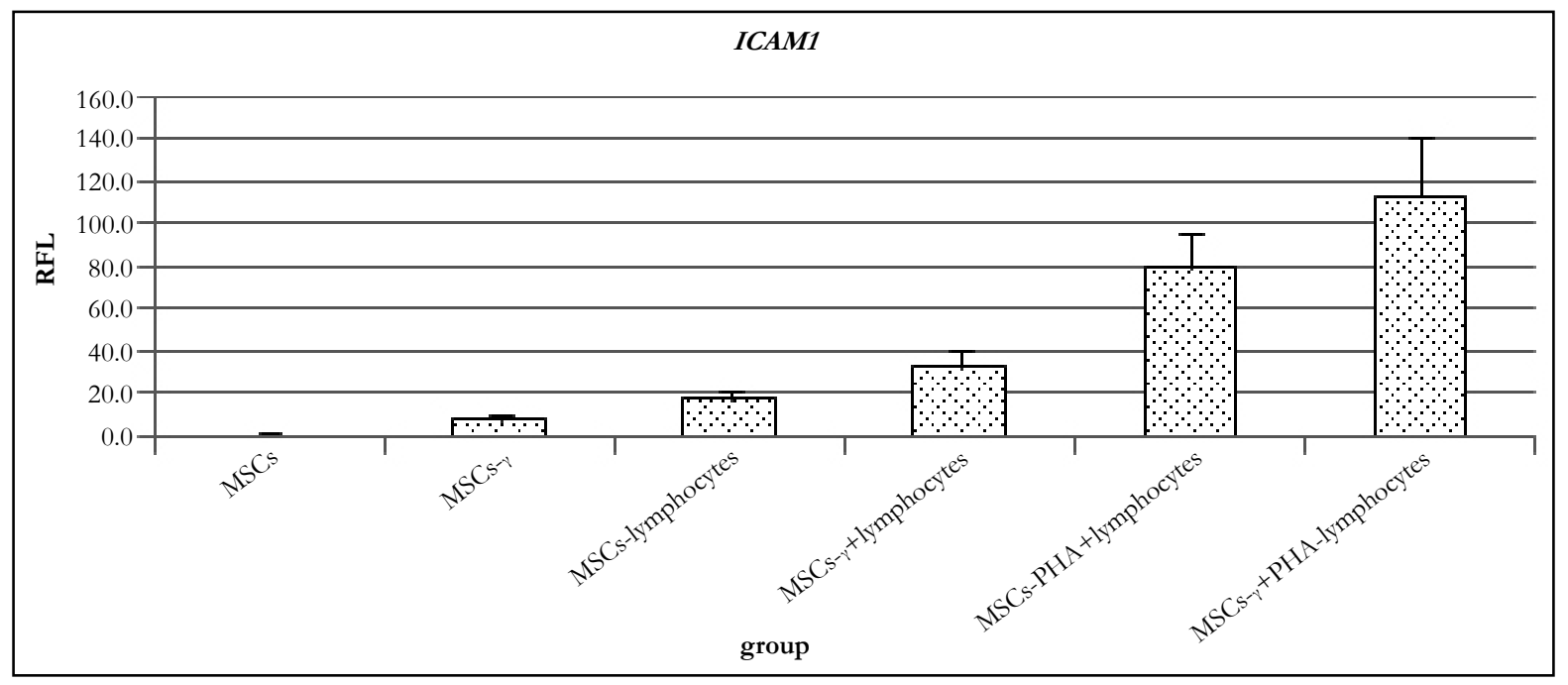

Figure 6. Alterations in REL of IDO1 in MSCs.
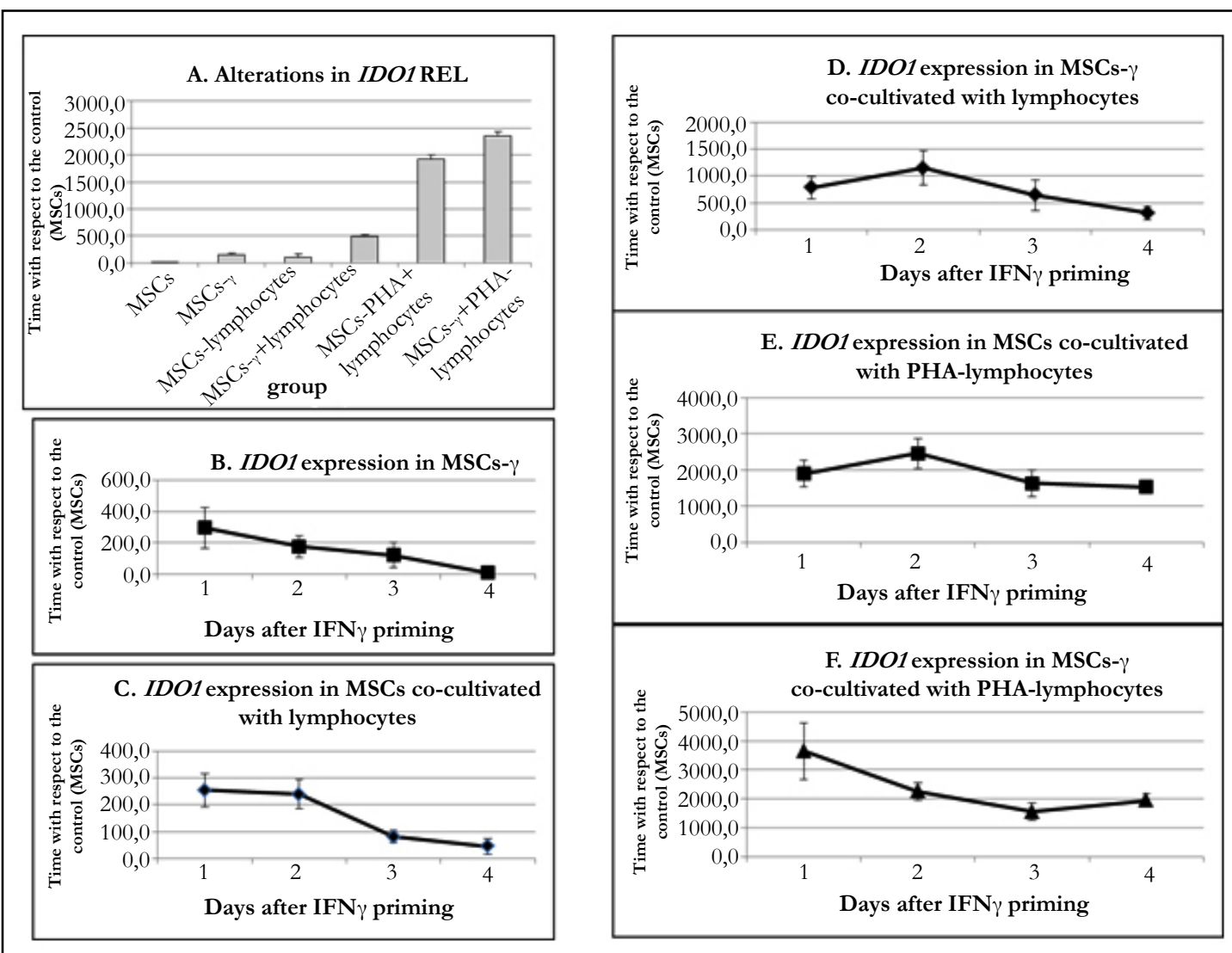

During culture, the REL of IDO1 decreased in all variants of experiments. However, if the effect was strong enough (IFN- $\gamma$ primed cells or PHA-lymphocytes), on the $4^{\text {th }}$ day, the REL of IDO1 in MSCs remained very high, i.e., 450-2000 times higher than in control MSCs. Thus, in the case of cell therapy, it may be most effective to administer IFN- $\gamma$-activated MSCs on the first day after priming. The effect of the exposure to IFN- $\gamma$ in vitro explains the enhanced therapeutic activity of MSCs in the model of colitis [28], as well as their better activity after freezing and thawing [12].

It is known that MSCs secrete a complement inhibitor - complement factor $\mathrm{H}(\mathrm{CFH})[60,61]$. CFH inhibits complement activa- tion by limiting the activity of $\mathrm{C} 3$ and $\mathrm{C} 5$ convertases and by contributing to the inactivation of $\mathrm{C} 3 \mathrm{~b}$ and $\mathrm{C} 4 \mathrm{~b}$. MSCs constitutively produce $C F H$, and this production is increased under the action of IFN- $\gamma$ and TNF- $\alpha$ but not of IL-6 [57]. The REL of CFH also increased in MSCs- $\gamma$ and MSCs co-cultured with lymphocytes (Figure 7A).

No significant changes in the REL of $\mathrm{CFH}$ in the MSCs were observed during culture. In both MSCs- $\gamma$ and MSCs co-cultured with lymphocytes, the REL of CFH increased by 2-2.5 times (significant differences were detected only one day after IFN- $\gamma$ treatment and when co-cultured with lymphocytes; $\mathrm{p}=0.01$ and 0.001 , respectively). The addition of PHA-lymphocytes increased the 
expression of this gene by 3-4 times in comparison with control MSCs.

\section{PTGES expression}

The REL of PTGES did not change in the MSCs- $\gamma$ (Figure 7B). When MSCs were co-cultured with lymphocytes, the REL of PTGES increased by 2 times but not significantly. Co-culture of MSCs- $\gamma$ with lymphocytes increased the expression of this gene by 4 times $(\mathrm{p}=0.003)$. Co-culture of MSCs and MSCs- $\gamma$ with PHA-lymphocytes increased the REL of PTGES by 3.7 and 4.2 times, respectively (both $\mathrm{p}=0.03$ ).

The immunomodulatory properties of MSCs can be enhanced by various cytokines secreted by activated lymphocytes. The effects of these molecules on MSCs depends on the cytokine type and their concentrations [61]. At the functional level, it has been shown that the treatment of MSCs with IFN- $\gamma$ protects these cells from NK-cells by enhancing the synthesis of prostaglandin E2 [36]. Both priming with IFN- $\gamma$ [43] and interacting with lymphocytes [30] induce PTGES expression in MSCs.

\section{IL-6 expression}

It has been shown that MSCs produce IL- 6 , which promotes the inhibition of monocyte differentiation into antigen-presenting cells [33]. It is known that IFN- $\gamma$ treatment of MSCs for 4 days leads to an increase in the IL-6 expression level [66]. In our experiments, MSC priming with IFN- $\gamma$ did not affect the REL of IL- 6 (Figure 7C). When MSCs and MSCs- $\gamma$ were co-cultured with lym- phocytes, the REL of IL- 6 increased by almost 20 times $(\mathrm{p}=0.015$ and $\mathrm{p}=0.0005$, respectively). During culture, the REL of IL- 6 in MSCs decreased by 2 times. However, in the case of MSCs- $\gamma$, the REL of IL- 6 did not decrease. Co-culture of MSCs and MSCs- $\gamma$ with PHA-lymphocytes led to increases in the REL of IL-6 of 25 times, and in both cases, the REL was reduced by half by the $4^{\text {th }}$ day of co-culture.

Thus, the treatment of MSCs with IFN- $\gamma$ resulted in a stable REL of IL-6 without any decrease after these cells interacted with lymphocytes. This fact should be taken into the account for improving the immunomodulatory functions of MSCs injected into an organism.

\section{CSF1 expression}

The REL of CSF1 increased in the MSCs- $\gamma$ by 2 times $(\mathrm{p}=0.05)$ (Figure 7D). Incubation of MSCs with lymphocytes increased the REL of CSF1 by 4 times ( $\mathrm{p}=0.02)$. Co-culture of MSCs- $\gamma$ with lymphocytes increased the REL of CSF1 by 7 times $(\mathrm{p}=0.004)$. When MSCs and MSCs- $\gamma$ were co-cultured with PHA-lymphocytes, the REL of CSF1 increased by 10 times ( $\mathrm{p}=0.04$ and 0.05 , respectively) and decreased after 3 days by 2 times compared to the first and second days of co-culture $(p=0.0009)$. In all other cases, the increased REL of this factor did not depend on the duration of co-culture.

In summary, the data suggest that IFN- $\gamma$ stabilized and activated the expression of CSF1, which is involved in the immunomodulatory properties of MSCs.

Figure 7. Alterations in REL of $C F H, P T G E S, I L-6$ and $C S F 1$ in MSCs.

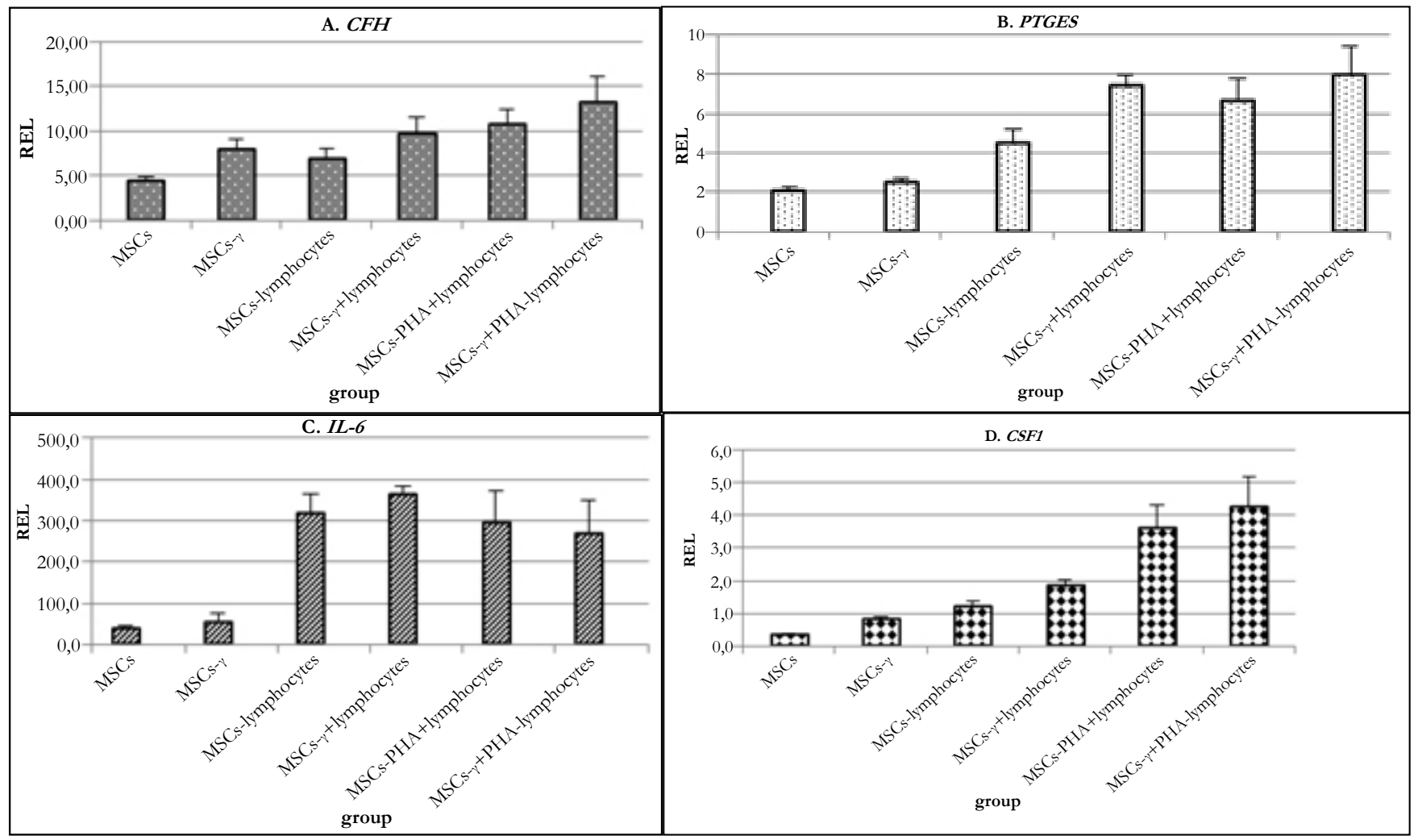

A. Alterations in REL of $C F H$

B. Alterations in REL of PTGES

C. Alterations in REL of $I L-6$

D. Alterations in REL of CSF1 
The data clearly identified significant changes in MSCs after lymphocyte interaction and following IFN- $\gamma$ treatment.

It is assumed that the immunomodulatory properties of MSCs are not constitutive and can be induced by inflammatory factors secreted by activated lymphocytes. The immunomodulatory effect of MSCs depends on the type and concentration of cytokines, indicating the plasticity of these cells [61]. This study simulates the interaction of MSCs with lymphocytes that possibly occurs in vivo.

When MSCs interact with activated lymphocytes, HLA-DR begins to be expressed on their surface more extensively than after IFN- $\gamma$ priming. This fact is very important for the selection of the source of MSCs for the treatment of autoimmune diseases and a GVHD. The preference of autologous MSCs is obvious in the first case, and MSCs obtained from hematopoietic stem cells of the donor should be used in the second case. According to this study, the immune privilege of MSCs disappears within 2 days of their interaction with the recipient's lymphocytes. Moreover, the data suggest rapid elimination of MSCs from the organism, as confirmed by the inability to detect MSCs 2 weeks after their administration $[2,19]$. IFN- $\gamma$ stabilized the MSCs, and the proportion of viable cells was significantly higher in MSCs- $\gamma$ after 4 days of co-culture with lymphocytes. Perhaps the effect of IFN- $\gamma$ on the stabilization of MSCs also helps to maintain their immunomodulatory properties after cryopreservation [12]. The changes in the properties of MSCs did not strongly depend on the source of IFN- $\gamma$ (exogenous or IFN- $\gamma$ secreted by lymphocytes). The level of ICAM-1 expression on the MSCs increased, which led to elevation of their adhesive properties, and the decrease in CD90 expression may indicate a change in the differentiation state of the cells in the population. Both the IFN- $\gamma$ treatment and lymphocyte interaction induced increases in the RELs of factors involved in immunomodulation (IDO1, CFH, PTGES, IL6, and CSF1). However, we have not obtained any evidence to support the hypothesis that the treatment of MSCs with IFN- $\gamma$ enhances their immunomodulatory properties during intravenous administration. Interaction with the recipient's lymphocytes causes the same changes in the main properties of MSCs as IFN- $\gamma$ priming. However, the treatment with IFN- $\gamma$ stabilizes the MSCs while maintaining their viability.

Based on the results of this work, the following are recommended for cell-based therapies: 1. MSCs obtained from the hematopoietic cells donor or autologous 2. short-term pretreatment of the MSCs with IFN- $\gamma$ prior to their use in cell-based therapies for immunomodulation.

\section{Acknowledgments \& Declarations}

The study was supported by a grant from the Russian Science Foundation, Project № 16-15-00102.

\section{References}

[1]. Akiyama K, Chen C, Wang D, Xu X, Qu C, et al. (2012) Mesenchymalstem-cell-induced immunoregulation involves FAS-ligand-/FAS-mediated T cell apoptosis. Cell Stem Cell. 10(5): 544-55.

[2]. Allers C, Sierralta WD, Neubauer S, Rivera F, Minguell JJ, et al. (2004) Dynamic of Distribution of Human Bone Marrow-Derived Mesenchymal Stem Cells After Transplantation into Adult Unconditioned Mice. Trans- plantation. 78(4): 503-508

[3]. Ankrum JA, Ong JF, Karp JM (2014) Mesenchymal stem cells: immune evasive, not immune privileged. Nat Biotechnol. 32(3): 252-60.

[4]. Augello A, Tasso R, Negrini SM, Amateis A, Indiveri F, et al. (2005) Bone marrow mesenchymal progenitor cells inhibit lymphocyte proliferation by activation of the programmed death 1 pathway. Eur J Immunol. 35(5): 1482-90.

[5]. Benvenuto F, Voci A, Carminati E, Gualandi F, Mancardi G, et al. (2015) Human mesenchymal stem cells target adhesion molecules and receptors involved in T cell extravasation. Stem Cell Res Ther. 6: 245 .

[6]. Bernot D, Peiretti F, Canault M, Juhan-Vague I, Nalbone G (2005) Upregulation of TNF-?-induced ICAM-1 surface expression by adenylate cyclase-dependent pathway in human endothelial cells. J Cell Physiol. 202(2): 434-441.

[7]. Le Blanc K, Rasmusson I, Götherström C, Seidel C, Sundberg B, et al. (2004) Mesenchymal stem cells inhibit the expression of CD25 (interleukin-2 receptor) and CD38 on phytohaemagglutinin-activated lymphocytes. Scand J Immunol. 60(3): 307-315.

[8]. Le Blanc K, Tammik C, Rosendahl K, Zetterberg E, Ringdén O (2003) HLA expression and immunologic properties of differentiated and undifferentiated mesenchymal stem cells. Exp Hematol. 31(10): 890-896.

[9]. Bruno S, Deregibus MC, Camussi G (2015) The secretome of mesenchymal stromal cells: Role of extracellular vesicles in immunomodulation. Immunol Lett. 168(2): 154-158.

[10]. Cao W, Cao K, Cao J, Wang Y, Shi Y (2015) Mesenchymal stem cells and adaptive immune responses. Immunol Lett. 168(2): 147-153.

[11]. Chan WK, Sik-Yin Lau A, Chun-Bong Li J, Ka-Wai Law H, Lau YL, et al. (2008) MHC expression kinetics and immunogenicity of mesenchymal stromal cells after short-term IFN- $\gamma$ challenge. Exp Hematol. 36(11): 15451555.

[12]. Chinnadurai R, Copland IB, Garcia MA, Petersen CT, Lewis CN, et al. (2016) Cryopreserved Mesenchymal Stromal Cells Are Susceptible to TCell Mediated Apoptosis Which Is Partly Rescued by IFN $\gamma$ Licensing. Stem Cells. 34(9): 2429-2442.

[13]. Chomczynski P, Sacchi N (1987) Single-step method of RNA isolation by acid guanidinium thiocyanate-phenol-chloroform extraction. Anal Biochem. 162(1): 156-9.

[14]. Consentius C, Reinke P, Volk H-D (2015) Immunogenicity of allogeneic mesenchymal stromal cells: what has been seen in vitro and in vivo ? Regen Med. 10(3): 305-315.

[15]. Dinarello CA (2011) Interleukin-1 in the pathogenesis and treatment of inflammatory diseases. Blood. 117(14): 3720-32.

[16]. Dominici M, Le Blanc K, Mueller I, Slaper-Cortenbach I, Marini F, et al. (2006) Minimal criteria for defining multipotent mesenchymal stromal cells. The International Society for Cellular Therapy position statement. Cytotherapy. 8(4): 315-7.

[17]. Duijvestein M, Wildenberg ME, Welling MM, et al. (2011) Pretreatmen with interferon- $\gamma$ enhances the therapeutic activity of mesenchymal stromal cells in animal models of colitis. Stem Cells. 29(10): 1549-58.

[18]. Ehninger A, Trumpp A (2011) The bone marrow stem cell niche grows up: mesenchymal stem cells and macrophages move in. J Exp Med. 208(3): 421-8.

[19]. Gao J, Dennis JE, Muzic RF, Lundberg M, Caplan a I (2001) The dynamic in vivo distribution of bone marrow-derived mesenchymal stem cells after infusion. Cells Tissues Organs. 169(1): 12-20.

[20]. Giuliani M, Poggi A, A BG, Jj L. (2014) IFNGamma Priming Protects Fetal and Embryonic MSC from NK Cell-Mediated Killing and Improves their Immunosuppressive Properties : Role of Activating and Inhibitory Receptors. J Cell Sci Ther. 5: 164.

[21]. Introna M, Rambaldi A (2015) Mesenchymal stromal cells for prevention and treatment of graft-versus-host disease: successes and hurdles. Curr Opin Organ Transplant. 20(1): 72-8.

[22]. Janeway CA Jr, Travers P, Walport M, MJ Shlomchik (2001) Immunobiology: The Immune System in Health and Disease. ( 5 th edn), Garland Science, New York.

[23]. Jiang D, Muschhammer J, Qi Y, et al (2016) Suppression of NeutrophilMediated Tissue Damage-A Novel Skill of Mesenchymal Stem Cells. Stem Cells. 34(9): 2393-2406.

[24]. Jones E, McGonagle D (2008) Human bone marrow mesenchymal stem cells in vivo. Rheumatology (Oxford). 47(2): 126-31.

[25]. Kawai M, Rosen CJ (2010) PPAR $\gamma$ : a circadian transcription factor in adipogenesis and osteogenesis. Nat Rev Endocrinol. 6(11): 629-36.

[26]. Kumar A, Bhanja A, Bhattacharyya J, Jaganathan BG (2016) Multiple roles of CD90 in cancer. Tumor Biol. 37(9): 11611-11622.

[27]. Kuzmina LA, Petinati NA, Parovichnikova EN, et al. (2012) Multipotent Mesenchymal Stromal Cells for the Prophylaxis of Acute Graft-versus-Host Disease - A Phase II Study. Stem Cells Int. 
[28]. Li N, Feugier P, Serrurrier B, Latger-Cannard V, Lesesve JF, Stoltz JF, et al. (2007) Human mesenchymal stem cells improve ex vivo expansion of adult human CD34+ peripheral blood progenitor cells and decrease their allostimulatory capacity. Exp Hematol. 35(3): 507-515.

[29]. Luk F, de Witte SFH, Bramer WM, Baan CC, Hoogduijn MJ (2015) Efficacy of immunotherapy with mesenchymal stem cells in man: a systematic review. Expert Rev Clin Immunol. 11(5): 617-36.

[30]. Madrigal M, Rao KS, Riordan NH (2014) A review of therapeutic effects of mesenchymal stem cell secretions and induction of secretory modification by different culture methods. J Transl Med. 12(1): 260.

[31]. Majumdar MK, Keane-Moore M, Buyaner D, Hardy WB, Moorman MA, et al. Characterization and functionality of cell surface molecules on human mesenchymal stem cells. J Biomed Sci. 10(2): 228-41.

[32]. Meisel R, Zibert A, Laryea M, Göbel U, Däubener W, et al. (2004) Human bone marrow stromal cells inhibit allogeneic T-cell responses by indoleamine 2,3-dioxygenase-mediated tryptophan degradation. Blood. 103(12): 4619-21.

[33]. Melief SM, Geutskens SB, Fibbe WE, Roelofs H (2013) Multipotent stromal cells skew monocytes towards an anti-inflammatory interleukin10-producing phenotype by production of interleukin-6. Haematologica. 98(6): 888-895.

[34]. Moraes DA, Sibov TT, Pavon LF, et al (2016) A reduction in CD90 (THY1) expression results in increased differentiation of mesenchymal stromal cells. Stem Cell Res Ther. 7(1): 97.

[35]. Najar M, Raicevic G, Jebbawi F, De Bruyn C, Meuleman N, et al. (2012) Characterization and functionality of the CD200-CD200R system during mesenchymal stromal cell interactions with T-lymphocytes. Immunol Lett. 146(1-2): 50-6.

[36]. Noone C, Kihm A, English K, O’Dea S, Mahon BP (2013) IFN- $\gamma$ stimulated human umbilical-tissue-derived cells potently suppress NK activation and resist NK-mediated cytotoxicity in vitro. Stem Cells Dev. 22(22): 3003-14.

[37]. Oates JC, Reilly CM, Crosby MB, Gilkeson GS (2002) Peroxisome proliferator-activated receptor gamma agonists: potential use for treating chronic inflammatory diseases. Arthritis Rheum. 46(3): 598-605.

[38]. Opitz C a., Litzenburger UM, Lutz C, et al. (2009) Toll-like receptor engagement enhances the immunosuppressive properties of human bone marrow-derived mesenchymal stem cells by inducing indoleamine-2,3-dioxygenase-1 via Interferon-b and protein kinase R. Stem Cells. 27(4): 909-919.

[39]. Petinati NA, Shipunova IN, Bigildeyev AE, Kuz'mina LA, Momotyuk KS, et al. (2012) Analysis of expression of genes involved in immune response modulation in silent multipotent mesenchymal stromal cells. Bull Exp Biol Med. 153(2): 244-8.

[40]. Polchert D, Sobinsky J, Douglas G, et al (2008) IFN-gamma activation of mesenchymal stem cells for treatment and prevention of graft versus host disease. Eur J Immunol. 38(6): 1745-55.

[41]. Pourgholaminejad A, Aghdami N, Baharvand H, Moazzeni SM (2016) The effect of pro-inflammatory cytokines on immunophenotype, differentiation capacity and immunomodulatory functions of human mesenchymal stem cells. Cytokine. 85: 51-60.

[42]. Prasanna SJ, Gopalakrishnan D, Shankar SR, Vasandan AB (2010) ProInflammatory Cytokines, IFN $\gamma$ and $\mathrm{TNF} \alpha$, Influence Immune Properties of Human Bone Marrow and Wharton Jelly Mesenchymal Stem Cells Differentially. PLoS One. 5(2): e9016.

[43]. Prockop DJ (2013) Concise Review: Two negative feedback loops place mesenchymal stem/stromal cells at the center of early regulators of inflammation. Stem Cells. 31(10): 2042-2046.

[44]. Prockop DJ, Prockop SE, Bertoncello I (2014) Are Clinical Trials With Mesenchymal Stem/Progenitor Cells too Far Ahead of the Science? Lessons From Experimental Hematology. Stem Cells. 32(12): 3055-3061.

[45]. Qiao R-Y, Bai H, Wang C-B, Ou J-F, Zhang H-Y, et al. (2012) [Effects of interferon- $\gamma$ on expression of adhesion molecules in human umbilical cord mesenchymal stromal cells]. Zhongguo shi yan xue ye xue za zhi. 20(5): $1195-9$.
[46]. Rege TA, Hagood JS (2006) Thy-1, a versatile modulator of signaling affecting cellular adhesion, proliferation, survival, and cytokine/growth factor responses. Biochim Biophys Acta. 1763(10): 991-9.

[47]. Ringden O, Le Blanc K (2011) Mesenchymal stem cells for treatment of acute and chronic graft-versus-host disease, tissue toxicity and hemorrhages. Best Pract Res Clin Haematol. 24(1): 65-72.

[48]. Schmittgen TD, Livak KJ (2008) Analyzing real-time PCR data by the comparative CT method. Nat Protoc. 3(6): 1101-1108.

[49]. Selmani Z, Naji A, Zidi I, et al (2008) Human leukocyte antigen-G5 secretion by human mesenchymal stem cells is required to suppress $\mathrm{T}$ lymphocyte and natural killer function and to induce CD4+CD25highFOXP3+ regulatory T cells. Stem Cells. 26(1): 212-22.

[50]. Sheng H, Wang Y, Jin Y, et al (2008) A critical role of IFNgamma in priming MSC-mediated suppression of $\mathrm{T}$ cell proliferation through up-regulation of B7-H1. Cell Res. 18(8): 846-57.

[51]. Sibov TT, Severino P, Marti LC, et al (2012) Mesenchymal stem cells from umbilical cord blood: parameters for isolation, characterization and adipogenic differentiation. Cytotechnology. 64(5): 511-521.

[52]. Sivanathan KN, Gronthos S (2014) Interferon-Gamma Modification of Mesenchymal Stem Cells : Implications of Autologous and Allogeneic Mesenchymal Stem Cell Therapy in Allotransplantation. 10(3): 351-75 DOI : 10.1007/s12015-014-9495-2.

[53]. Sivanathan KN, Rojas-Canales D, Hope CM, Krishnan R, Carroll RP, et al (2015) Interleukin-17A induced human Mesenchymal Stem Cells are superior modulators of immunological function. Stem Cells. 33(9): 2850- 2863.

[54]. Song Y, Lin Q, Zheng J, Zhu X, Yang S (2014) [PPAR- $\gamma$ agonist inhibits the expressions of chemokines induced by IFN- $\gamma$ and TNF- $\alpha$ in renal tubular epithelial cells]. Xi Bao Yu Fen Zi Mian Yi Xue Za Zhi. 30(7): 673-6.

[55]. Spees JL, Lee RH, Gregory CA (2016) Mechanisms of mesenchymal stem/ stromal cell function. Stem Cell Res Ther. 7(1): 125

[56]. Svinareva DA, Shipunova IN, Olshanskaya YV, Momotyuk KS, Drize NI, et al. (2010) The basic properties of bone marrow mesenchymal stromal cells from donors: Superficial markers. Ter Arkh. 82(7): 52-56. https://www. ncbi.nlm.nih.gov/pubmed/20853610

[57]. Tu Z, Li Q, Bu H, Lin F (2010) Mesenchymal stem cells inhibit complement activation by secreting factor H. Stem Cells Dev. 19(11): 1803-9.

[58]. Vaquero C, Sancéau J, Catinot L, Andreu G, Falcoff E, et al. (1982) Translation of mRNA from phytohemagglutinin-stimulated human lymphocytes: characterization of interferon mRNAs. J Interferon Res. 2(2): 217-28.

[59]. Vestweber D (2015) How leukocytes cross the vascular endothelium. Nat Rev Immunol. 15(11): 692-704

[60]. Voswinkel J, Francois S, Simon J-M, Benderitter M, Gorin N-C, et al. (2013) Use of mesenchymal stem cells (MSC) in chronic inflammatory fistulizing and fibrotic diseases: a comprehensive review. Clin Rev Allergy Immunol. 45(2): 180-92.

[61]. Wang Y, Chen X, Cao W, Shi Y (2014) Plasticity of mesenchymal stem cells in immunomodulation: pathological and therapeutic implications. Nat Immunol. 15(11): 1009-1016.

[62]. Wu X, Wang W, Meng C, Yang S, Duan D, et al. (2013) Regulation of differentiation in trabecular bone-derived mesenchymal stem cells by $\mathrm{T}$ cell activation and inflammation. Oncol Rep. 30(5): 2211-9.

[63]. Xue Q, Luan X-Y, Gu Y-Z, et al (2010) The negative co-signaling molecule b7-h4 is expressed by human bone marrow-derived mesenchymal stem cells and mediates its T-cell modulatory activity. Stem Cells Dev. 19(1): 27-38.

[64]. Yang L, Froio RM, Sciuto TE, Dvorak AM, Alon R, et al. (2005) ICAM-1 regulates neutrophil adhesion and transcellular migration of TNF-alphaactivated vascular endothelium under flow. Blood. 106(2): 584-92.

[65]. Zhang Z, Han Y, Song J, Luo R, Jin X, et al. (2015) Interferon- $\gamma$ regulates the function of mesenchymal stem cells from oral lichen planus via indoleamine 2,3-dioxygenase activity. J Oral Pathol Med. 44(1): 15-27.

[66]. Zimmermann JA, Mcdevitt TC (2014) Pre-conditioning mesenchymal stromal cell spheroids for immunomodulatory paracrine factor secretion. Cytotherapy. 16(3): 331-345. 